\title{
Intracisternal delivery of NFкB-inducible scAAV2/9 reveals locoregional neuroinflammation induced by systemic kainic acid treatment
}

\author{
Olivier Bockstael ${ }^{1}$, Liliane Tenenbaum ${ }^{2}$, Deniz Dalkara ${ }^{3,4,5}$, Catherine Melas $^{1}$, Olivier De Witte ${ }^{1}$, \\ Marc Levivier $^{2}$ and Abdelwahed Chtarto ${ }^{1}$ * \\ ${ }^{1}$ Laboratory of Experimental Neurosurgery and Multidisciplinary Research Institute, Institut de Recherche Interdisciplinaire en Biologie Humaine et \\ Moléculaire - Université Libre de Bruxelles, Bruxelles, Belgium \\ ${ }^{2}$ Neurosurgery Service and Laboratory of Cellular and Molecular Neurotherapies, Department of Clinical Neuroscience, Lausanne University Hospital, \\ Lausanne, Switzerland \\ 3 INSERM, U968, Paris, France \\ ${ }^{4}$ Sorbonne Universités, UPMC Univ. Paris 06, UMR_S 968, Institut de la Vision, Paris, France \\ ${ }^{5}$ CNRS, UMR_7210, Paris, France
}

\section{Edited by:}

Erwan Bezard, Institut des Maladies Neurodegeneratives, France

\section{Reviewed by:}

Björn Spittau, Albert-LudwigsUniversity Freiburg, Germany Benjamin Dehay, Institute of Neurodegenerative Diseases - CNRS UMR5293, France

Bernard Laurent Schneider, École Polytechnique Fédérale de Lausanne, Switzerland

\section{*Correspondence:}

Abde/wahed Chtarto, Laboratory of Experimental Neurosurgery and Multidisciplinary Research Institute, Institut de Recherche Interdisciplinaire en Biologie Humaine et Moléculaire Université Libre de Bruxelles,

Bruxelles, Belgium

e-mail: achtarto@ulb.ac.be
We have previously demonstrated disease-dependent gene delivery in the brain using an $A A V$ vector responding to $N F_{K} B$ activation as a probe for inflammatory responses. This vector, injected focally in the parenchyma prior to a systemic kainic acid (KA) injection mediated inducible transgene expression in the hippocampus but not in the cerebellum, regions, respectively, known to be affected or not by the pathology. However, such a focal approach relies on previous knowledge of the model parameters and does not allow to predict the whole brain response to the disease. Global brain gene delivery would allow to predict the regional distribution of the pathology as well as to deliver therapeutic factors in all affected brain regions. We show that self-complementary AAV2/9 (scAAV2/9) delivery in the adult rat cisterna magna allows a widespread but not homogenous transduction of the brain. Indeed, superficial regions, i.e., cortex, hippocampus, and cerebellum were more efficiently transduced than deeper regions, such as striatum, and substantia nigra. These data suggest that viral particles penetration from the cerebrospinal fluid (CSF) into the brain is a limiting factor. Interestingly, AAV2/9-2YF a rationally designed capsid mutant (affecting surface tyrosines) increased gene transfer efficiency approximately fivefold. Neurons, astrocytes, and oligodendrocytes, but not microglia, were transduced in varying proportions depending on the brain region and the type of capsid. Finally, after a single intracisternal injection of scAAV2/9-2YF using the NFKB-inducible promoter, KA treatment induced transgene expression in the hippocampus and cortex but not in the cerebellum, corresponding to the expression of the CD11b marker of microglial activation. These data support the use of disease-inducible vectors administered in the cisterna magna as a tool to characterize the brain pathology in systemic drug-induced or transgenic disease models. However, further improvements are required to enhance viral particles penetration into the brain.

Keywords: AAV, neuroinflammation, inducible vector, cisterna magna, cerebrospinal fluid

\section{INTRODUCTION}

Increasing evidence suggests that global brain neuroinflammation is a key factor in the progression of neurodegenerative diseases (Dexter and Jenner, 2013). However, a controlled inflammatory response can also be beneficial for brain repair (London et al., 2013). Therefore, approaches aiming at the reduction of neuroinflammation should use conditional expression of the interfering factor in excessive stress conditions (Brown and Neher, 2014). A reduction of toxic intracerebral conditions such as neuroinflammation could possibly be achieved through global brain gene delivery while avoiding the risk of surgery-related local inflammatory reactions.
Intraperitoneal injection of kainic acid (KA), a model of temporal lobe epilepsy (Williams et al., 2009), is known to induce a locoregional inflammatory response in the hippocampus and in the cortex (Somera-Molina et al., 2009) but not in the cerebellum (Rong and Baudry, 1996).

In order to specifically express disease-interfering genes it is desirable to use a disease-regulated vector providing a similar pattern of transgene expression.

Gene delivery to the whole brain can be achieved through intravenous administration followed by trans-blood-brain barrier (BBB) passage (Duque et al., 2009; Foust et al., 2009; Mccarty et al., 2009; Fu et al., 2011; Gray et al., 2011) or by intra-cerebro- 
spinal fluid (CSF) administration followed by passage through the CSF-brain barrier (Fu et al., 2003, 2010; Samaranch et al., 2012, 2013; Gray et al., 2013). The BBB and CSF-brain barriers being of different nature, the regions and cell types transduced as well as the immune reaction toward the viral capsids and transgene products are expected to be different. Indeed, intracisternal injections are characterized by a reduced immune response and maintenance of transgene expression despite pre-existing immunity as compared to intravenous injections. However, Samaranch and collaborators (Samaranch et al., 2012) showed that, at least in monkeys, pre-existing anti-AAV antibodies abrogated brain transduction following delivery into the cisterna magna CSF. Another advantage of intracisternal versus intravenous injections is the lower biodistribution in peripheral organs (Gray et al., 2013).

Intracisternal injections of self-complementary (sc) AAV2 in juvenile mice combined with systemic mannitol administration (Fu et al., 2003, 2010), of scAAV2/9 and scAAV2/7 in non-human primates (Mccarty et al., 2009; Gray et al., 2011, 2013; Samaranch et al., 2013) as well as of scAAV2/9 and scAAV2/1 in the cat (Bucher etal., 2013) have been described with reports of neurons and astrocytes being transduced in the brain and spinal cord.

Largely documented models hypothesize that the CSF produced in the choroid plexus of the lateral ventricles, travels to the cisterna magna, then to the subarachnoid space and further moves into the brain in the perivascular VirchowRobin space surrounding penetrating cerebral arteries. Consistently with this model, large molecules tracers $(2000 \mathrm{kD})$ injected in the cisterna magna accumulate along perivascular spaces mainly in superficial layers (cortex; Iliff et al., 2012). As predicted by these models, AAV-mediated gene delivery after intracisternal injection was maximal in regions close to Virchow-Robin subarachnoid space (cerebellum and cortex) and reduced in deeper regions (Samaranch et al., 2013).

It has recently been shown that gene delivery to the retina and the brain of neonatal mice through systemic intravenous injection of AAV2/9 was enhanced by a capsid mutant in which two tyrosine residues presumably involved in capsid ubiquitination were replaced by phenylalanines (Dalkara et al., 2012). Here we investigated whether such mutations could also enhance gene delivery to the brain after intra-CSF delivery.

We compared the distribution and cell-type specificity of gene transfer into the brain after intracisternal injection of scAAV2/9 and the 2YF capsid mutant in the adult rat brain. With both vectors, gene transfer was prominent in the cerebellum and in the cortex with neurons, astrocytes and oligodendrocytes, but not microglia, being targeted in different proportions. The 2YF capsid mutant affected both the global efficiency and the proportions of neurons and glial cells.

We showed that, as expected, intraperitoneal KA injection induced transgene expression from a cisternally injected NFкBinducible scAAV2/9-2YF vector in the cortex and hippocampus but not in the cerebellum.

Pathology-inducible gene transfer could be used to probe pathological events as well as for regulated therapeutic gene delivery specifically in diseased brain regions.

\section{MATERIALS AND METHODS PLASMIDS}

The pSC-NF8-d1-EGFP plasmid containing eight NFkB responsive elements (two blocks of four consensus sequences, separated by $16 \mathrm{bps}$ ) fused to a minimal CMV promoter has been previously described (Chtarto et al., 2013).

The pHpaI-EGFP self-complementary AAV (scAAV) vector was a kind gift from D. McCarty and R. J. Samulski (Mccarty et al., 2003).

The pAAV2/9 and pAd Delta F6 were a kind gift from the Pen Vector Core (University of Pennsylvania). pAAV2/9-2YF was previously described (Dalkara et al., 2012).

\section{VIRAL PRODUCTION}

To produce recombinant scAAV2/9-CMV-EGFP, scAAV2/9-2YFCMV-EGFP and scAAV2/9-NF8-d1-EGFP viral stocks, HEK-293T cells $\left(5.0 \times 10^{6}\right.$ cells seeded on $10 \mathrm{~cm}$ plates) were co-transfected using polyethylenimine (PEI) from Polysciences, using a 5:1 (v:w) PEI:DNA ratio and a 2:3:5 molar ratio of vector plasmid, pAd plasmid and pAAV2/9 or pAAV2/9 2YF packaging plasmid expressing the AAV viral genes (rep gene from AAV serotype 2 and cap gene from AAV serotype 9 or from the 2 YF tyrosine mutant). Fifty hours post-transfection, the medium was discarded and the cells were harvested by low-speed centrifugation and resuspended in Tris $\mathrm{pH}$ 8.0, NaCl $0.1 \mathrm{M}$. After 5 cycles of freezing/thawing, the lysate was clarified by $30 \mathrm{~min}$ centrifugation at $10000 \mathrm{~g}$, treated with benzonase $(50 \mu / \mathrm{ml})$ at $37^{\circ} \mathrm{C}$ for $30 \mathrm{~min}$, and centrifuged at $10000 \mathrm{~g}$ for $30 \mathrm{~min}$ to eliminate the residual debris. The virus was further purified by iodixanol gradient. Viral genomes (vg) were titrated by quantitative PCR using primers located in the SV40polyA sequence (forward primer: AGC AAT AGC ATC ACA AAT TTC ACA A; reverse primer: CCA GAC ATG ATA AGA TAC ATT GAT GAG TT; internal probe: 6FAM- AGC ATT TTT TTC ACT GCA TTC TAG TTG TGG TTT GTC-TAMRA). The PCR reaction included a pre-denaturing step of $10 \mathrm{~min}$ at $95^{\circ} \mathrm{C}$ followed by 40 cycles of the 2 following steps: $95^{\circ} \mathrm{C}$ for $15 \mathrm{~s}$ and $60^{\circ} \mathrm{C}$ for $1 \mathrm{~min}$. (Lock et al., 2010). Titers were $1,4 \times 10^{13} \mathrm{vg} / \mathrm{ml}, 2.0 \times 10^{13}$ $\mathrm{vg} / \mathrm{ml}$ and $5,8 \times 10^{12} \mathrm{vg} / \mathrm{ml}$, respectively, for rAAV2/9-CMV-EGFP, rAAV2/9-2YF-CMV-EGFP, and rAAV2/9-2YF-NF8-d1-EGFP.

\section{ANIMALS}

For the analysis of constitutive transgene expression from the CMV promoter, adult female Wistar rats weighing approximately $200 \mathrm{~g}$ were used. For experiments with the KA model, adult male Sprague-Dawley rats weighing approximately $200 \mathrm{~g}$ were used. Animals were housed and treated according to the Belgian law. The protocols were in accordance with national rules on animal experiments and were approved by the Ethics Committee of the Faculty of Medicine of the "Université Libre de Bruxelles."

Animals were anesthetized with a mixture of ketamine (Imalgène 1000 , Merial; $100 \mathrm{mg} / \mathrm{kg}$ ) and xylazin (Rompun, Bayer; $10 \mathrm{mg} / \mathrm{kg}$ ) and placed on a Kopf stereotaxic frame (Kopf Instruments, Tujunga, CA, USA).

\section{Intracisternal injections}

The dura mater of the cisterna magna was made accessible by incision of the neck skin and gentle separation of the subcutaneous 
tissue and muscles under anesthesia as previously described (Liu and Duff, 2008). Ten minutes before virus injection a 25\% solution of D-mannitol (Sigma-Aldrich, M8429-100G) diluted in water and sterilized by filtration was injected intraperitoneally $(1 \mathrm{~g} / \mathrm{kg})$. Next, $10 \mu \mathrm{l}$ of viral suspension diluted at $10^{12} \mathrm{vg} / \mathrm{ml}$ was slowly injected under microscope using a silicone-coated glass capillary connected to a $50 \mu \mathrm{l}$ Hamilton syringe via polyethylene tubing. After viral infusion, the capillary was maintained in place for $2 \mathrm{~min}$ and then gently removed. The muscles were re-aligned, and the skin was sutured.

\section{Intraperitoneal KA injections}

Five weeks after the virus injection, induction of status epilepticus was performed as described earlier (Vermoesen et al., 2010). Briefly, consecutive intraperitoneal KA injections $(5 \mathrm{mg} / \mathrm{kg}$, diluted in PBS, Nanocs $\left.{ }^{\circledR}\right)$ were administered at 30 min interval. If a rat was nearing status epilepticus, half-doses $(2.5 \mathrm{mg} / \mathrm{kg})$ were given in order to reduce mortality. Control rats were injected with saline $(\mathrm{NaCl} 0.9 \%)$.

\section{Perfusions}

Animals were sacrificed one week after intraperitoneal KA injection and perfused intracardially first with saline, then with $4 \%$ paraformaldehyde (PF4). Brains were post-fixed for $24 \mathrm{~h}$ in PF4.

\section{IMMUNOHISTOCHEMISTRY}

For GFP staining, vibratome coronal brain sections $(50 \mu \mathrm{m})$ were immunostained using anti-GFP antibodies using peroxidase staining as previously described (Chtarto et al., 2013).

For CD11b staining, the procedure was the same except that mouse monoclonal anti-CD11b (1:500, Serotec, MorphoSys, Dusseldorf, Germany) was used as a primary antibody and goat antimouse IgG conjugated with HRP (Molecular Probes, Invitrogen, Carlsbad, CA, USA, from TSA kit) as a secondary antibody.

Densitometric analysis of the staining was performed using the image J software (NIH, USA).

\section{QUANTIFICATION METHOD}

Global brain transduction was quantified by two ways: cell density and staining proportion.

Cell density was assessed by counting cells on microscope pictures of known surface. All animals were photographed bilaterally on the same sections (same antero-posteriour level) at the same location (dorsal or lateral) under the same conditions (exposure, illumination) using an axioplan 2 microscope (Zeiss) with a 10X/0,30 plan NEOFLUAR objective (Zeiss). For cortex counting, 10 fields were photographed on five sections encompassing the cortex from frontal cortex to the beginning of the hippocampus. For hippocampus quantification, eight fields on four sections were photographed and counted.

Staining surface quantification was quantified on whole section mosaic pictures. One section out of six of all injected animals was DAB stained for GFP expression. All stained sections were photographed using the mosaic function of the Axiovision 4.8 software (Zeiss) on an imager M1 microscope (Zeiss) equipped with a 10X/0,3 EC plan NEOFLUAR objective (Zeiss). Section surface and stained surface were quantified in pixel using the threshold function of ImageJ software (NIH).

\section{IMMUNOFLUORESCENCE \\ GFP labeling}

Coronal brain sections $(50 \mu \mathrm{m})$ obtained using a vibratome (Leica Microsystems, Wetzlar, Germany) were incubated with a polyclonal rabbit anti-GFP (1:3000, Molecular Probes, Invitrogen, Carlsbad, CA, USA) followed by a biotin-streptavidin-cyanine 2 fluorescent labeling as previously described (Bockstael et al., 2012).

\section{GFP: NeuN and GFP:GFAP co-labelings}

For double immunofluorescence, the GFP labeling was combined with mouse monoclonal antibodies [anti-NeuN or anti-glial fibrillary acid protein (GFAP)] followed by donkey anti-mouse IgG coupled to cyanine 3 as previously described (Bockstael et al., 2012).

\section{GFP: Ibal co-labeling}

For GFP:IbaI double immunofluorescence, the above described GFP labeling was combined with goat anti-IbaI followed by a donkey anti-goat A.568 antibody as previously described (Bockstael et al., 2012).

\section{GFP: Olig2 co-labeling}

For GFP:Olig2 double immunofluorescence, a chicken monoclonal anti-GFP antibody and rabbit polyclonal anti-Olig2 IgG were used as previously described (Bockstael et al., 2012).

In order to better visualize the structure of the tissue, cerebellar sections were incubated in the Hoescht 33258 dye (Sigma-Aldrich) diluted at $1 \mu \mathrm{g} / \mathrm{ml}$ in TBS for $30 \mathrm{~min}$.

\section{CONFOCAL MICROSCOPY}

Co-labeling analysis was performed on pictures taken on at least three different sections using a LSM510 NLO multiphoton confocal microscope fitted on an Axiovert M200 inverted microscope equipped with C-Apochromat $40 \times / 1.2$ N.A. and $63 \times / 1.2$ N.A. water immersion objectives (Zeiss, Iena, Germany).

The $488 \mathrm{~nm}$ excitation wavelength of the Argon/2 laser, a main dichroic HFT 488 and a band-pass emission filter (BP500-550 nm) were used for selective detection of the green fluorochrome (Cy2, Alexa 488).

The $543 \mathrm{~nm}$ excitation wavelength of the HeNel laser, a main dichroic HFT 488/543/633 and a long-pass emission filter (BP565$615 \mathrm{~nm}$ ) were used for selective detection of the red fluorochrome (Cy3).

Optical sections, two microns thick, $512 \times 512$ pixels, were collected sequentially for each fluorochrome. Z-stacks with a focus step of one micron were collected.

The data-sets generated were merged and displayed with the Zen software (Zeiss, 2009) and exported in LSM image format.

Counting was performed with the Image 1.46 a software $(\mathrm{NIH}$, USA). Figures were prepared with Adobe Photoshop CS3 software. 


\section{STATISTICAL ANALYSIS}

All the statistical analysis was performed using the GraphPad Software. Results were expressed as mean \pm SD and statistical significance was evaluated with student $t$-test (to compare pairs of data sets). Differences were considered as significant when $p<0.05$.

\section{RESULTS}

GLOBAL BRAIN TRANSDUCTION AFTER INTRACISTERNAL DELIVERY OF ScAAV2/9 IS ENHANCED BY A RATIONALLY DESIGNED SURFACE TYROSINE CAPSID MUTATION

It has been shown that gene delivery to the retina and the brain of neonatal mice as well as newborn macaques through
FIGURE 1 | Locoregional brain transduction after intracisternal delivery of scAAV2/9-2YF-CMV-EGFP. The scAAV2/9-2YF-CMV-EGFP vector $\left(10^{10} \mathrm{vg}\right)$ was injected intracisternally. Five weeks after injection the rats $(n=5)$ were perfused and brain sections of $50 \mu \mathrm{m}$ were labeled with anti GFP antibodies. The picture corresponds to an antero-posterior succession of coronal rat brain sections. The mean volume of transduction is $8.14 \% \pm 3,3 \%$ of the whole brain. Scale bar: $5 \mathrm{~mm}$.
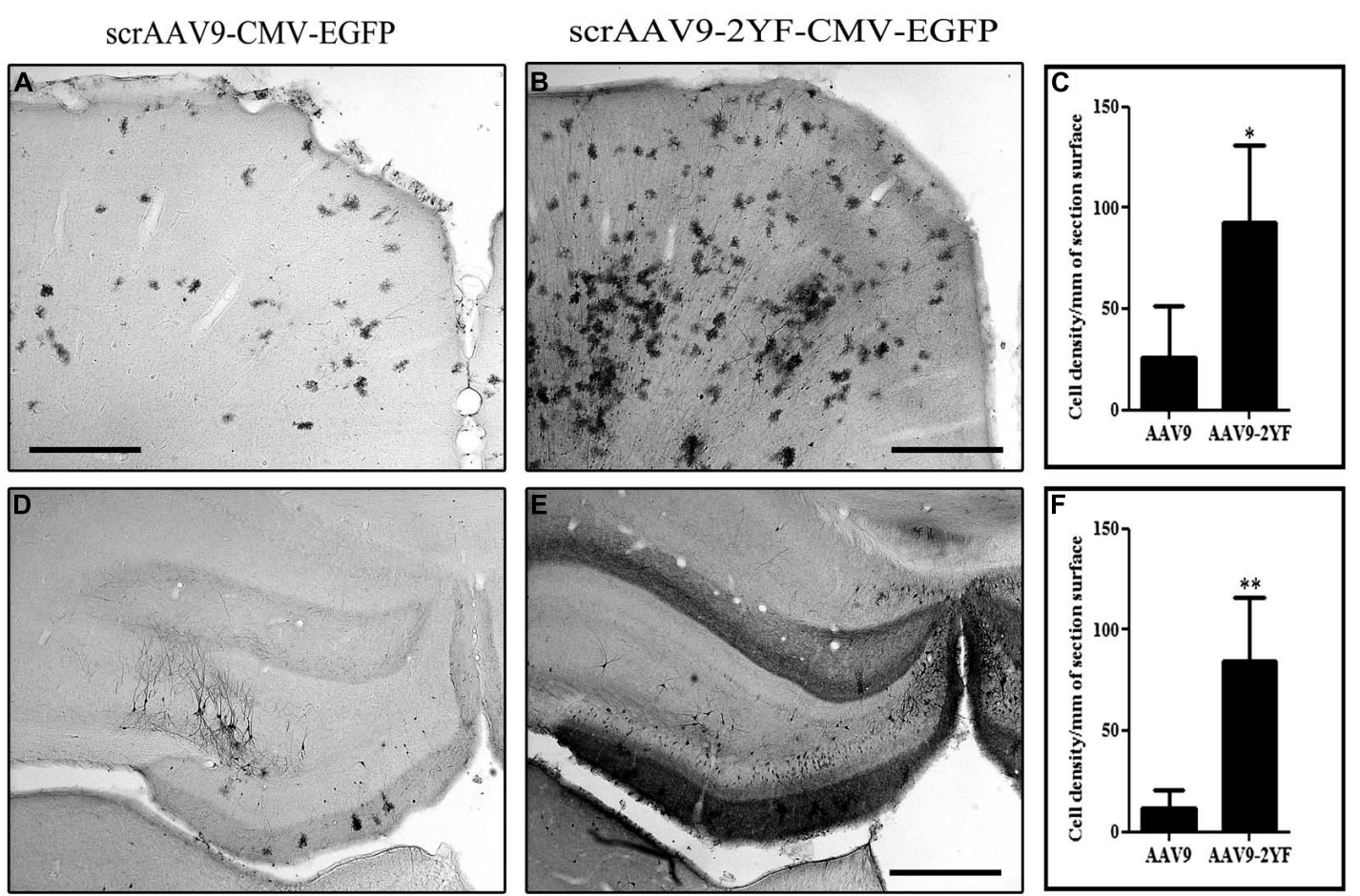

FIGURE 2 | Brain transduction after intracisternal delivery of scAAV2/9 is enhanced by mutation of two surface tyrosines. Recombinant scAAV2/9-CMV-EGFP and scAAV2/9-2YF-CMV-EGFP $\left(10^{10} \mathrm{vg}\right)$ were

injected intracisternally. Five weeks post-injection the rats were perfused and vibratome brain sections of $50 \mu \mathrm{m}$ were labeled with an anti GFP antibody. The pictures show representatives examples of GFP-labeling in the cortex (A,B) and of the hippocampus (D,E). Scale bar: $500 \mu \mathrm{m}$. The cell density in the cortex (C) and in the hippocampus (F) was evaluated as described in Section "Materials and Methods" and expressed as number of cells per square millimeter $\left(\mathrm{mm}^{2}\right)$. Data are expressed as mean $\pm \mathrm{SD}$. The results obtained show more GFP-marked cells $/ \mathrm{mm}^{2}$ of section surface in scAAV2/9-2YF compared to scAAV2/9 injected rats both in the cortex (C) $p=0,0395$, student $t$-test) and in the hippocampus (F; $p=0,0063$, student $t$-test). 


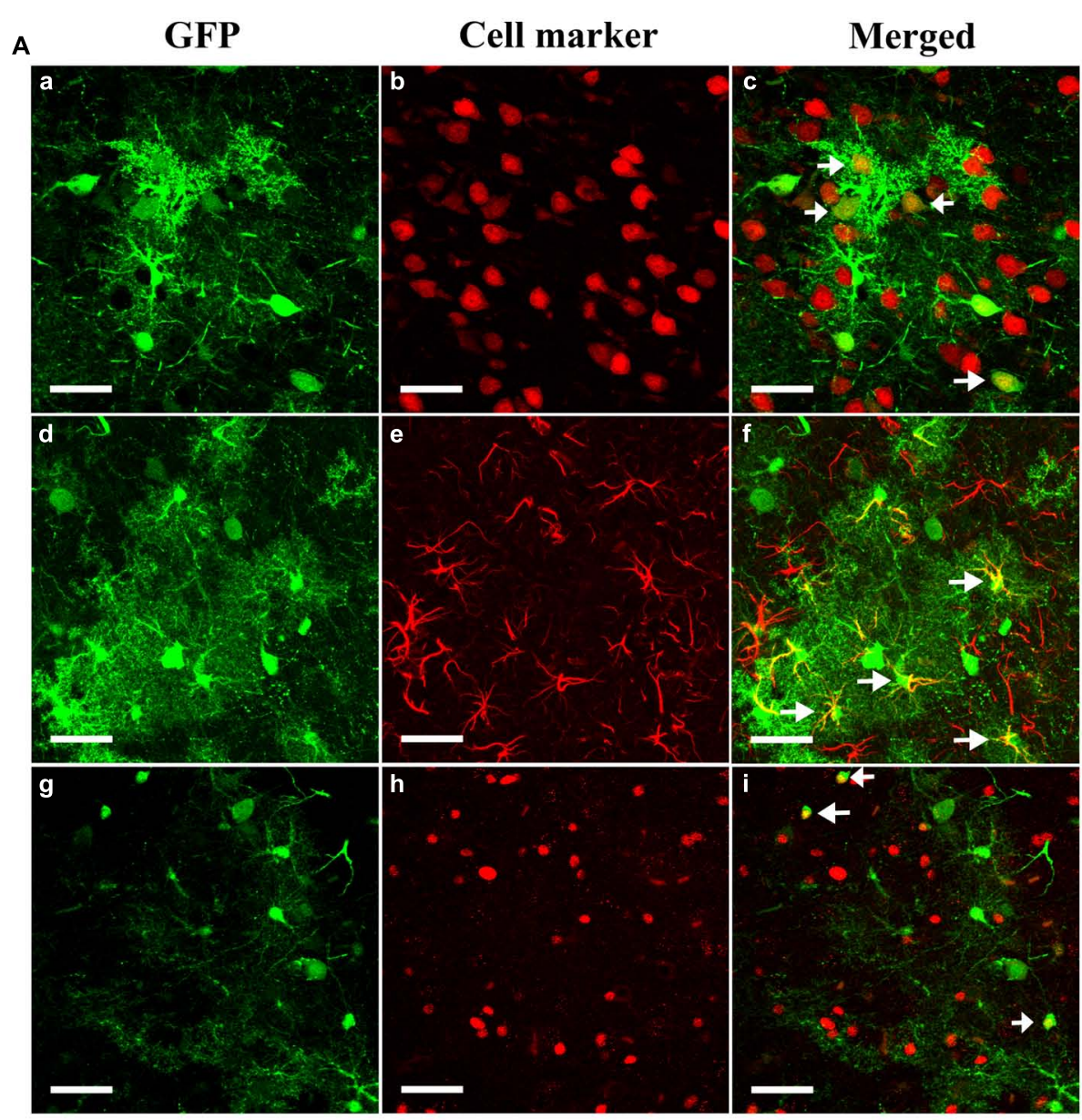

B

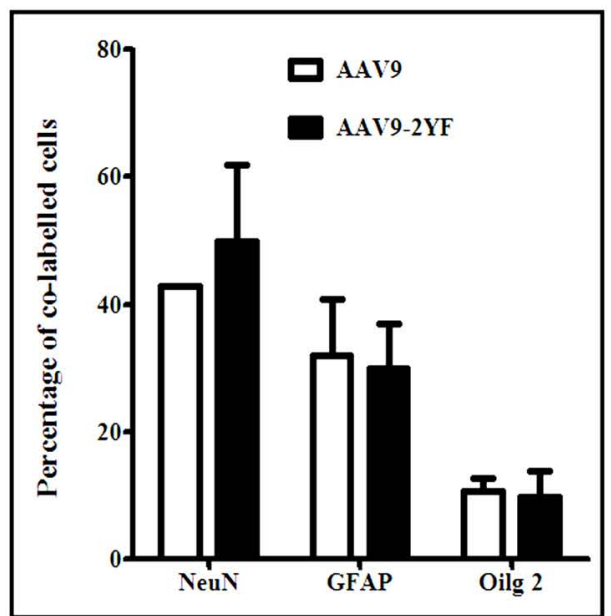

FIGURE 3 | Cellular specificity of ScAAV2/9-CMV-EGFP and scAAV2/9-2YF-CMV-EGFP in the cortex. (A) Five weeks after injection of scAAV2/9-CMV-EGFP $(n=5)$ or scAAV2/9-2YF-CMV-EGFP vector $(n=5)$ into the cisterna magna, brain sections were double-labeled with GFP (green fluorescence) and NeuN, GFAP or Olig2, (red fluorescence). Confocal pictures show GFP and cell-specific markers in double-labeled cells (yellow). GFP/NeuN, (a-c), GFP/GFAP, (d-f) and GFP/Olig2, (g-i). Arrowheads indicate double-labeled cells. Scale bar:
$40 \mu \mathrm{m}$. (B) the proportions of co-labeled cells were not significantly different for the two vectors for $\operatorname{NeuN}(p=0,3582$, student $t$-test), GFAP $(p=0,7971$, student $t$-test $))$ and Olig2 $(p=0,7622$, student $t$-test). Open columns, scAAV2/9-CMV-EGFP; solid columns,

scAAV2/9-2YF-CMV-EGFP. Data are expressed as means \pm SD. Analysis was performed using confocal microscopy and counting the number co-labeled cells on five sections per animal ( $n=3$ rats for GFP/NeuN, GFP/GFAP and GFP/Olig2). 

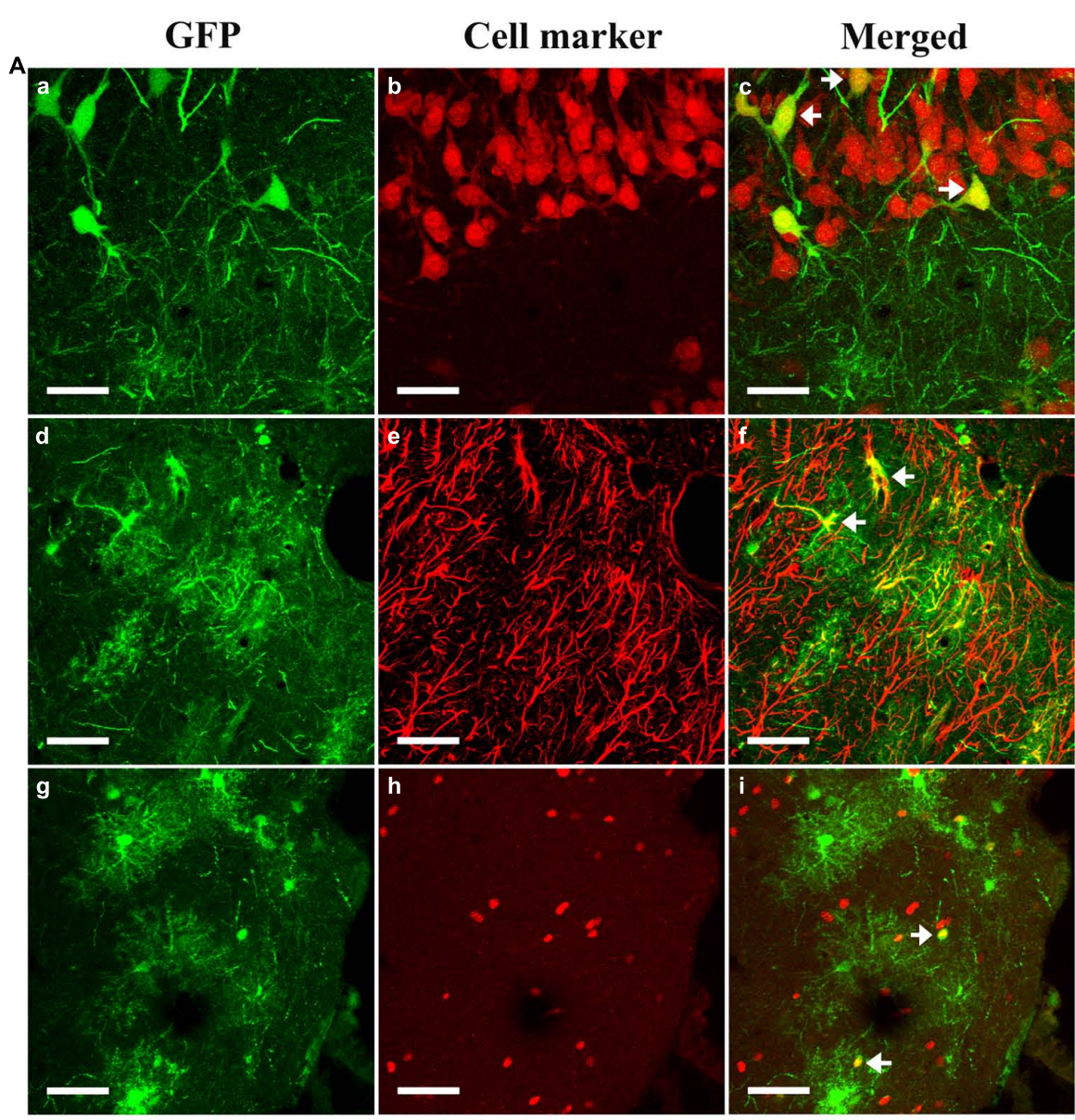

B

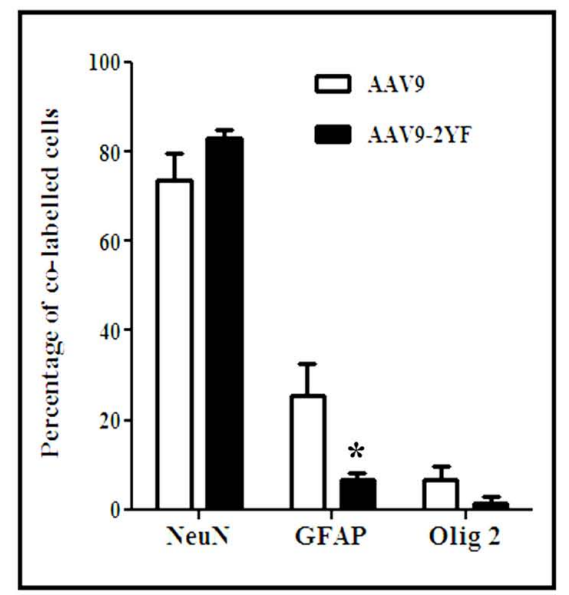

FIGURE 4 | Cellular specificity of scAAV2/9-CMV-EGFP and scAAV2/9-2YF-CMV-EGFP in the hippocampus. (A) Five weeks after injection of scAAV2/9-CMV-EGFP $(n=5)$ or ScAAV2/9-2YF-CMV-EGFP vector $(n=5)$ into the cisterna magna, brain sections were double-labeled with GFP (green fluorescence) and NeuN, GFAP, or Olig2 (red fluorescence). Confocal pictures show GFP and cell-specific markers in double-labeled cells (yellow). GFP/NeuN, (a-c), GFP/GFAP, (d-f) and GFP/Olig2, (g-i). Arrowheads indicate double-labeled cells. Scale bar:
$40 \mu \mathrm{m}$. (B) the proportions of co-labeled cells were not significantly different for the two vectors for $\operatorname{NeuN}(p=0,0602$, student $t$-test) and Olig2 ( $p=0,0500$, student $t$-test), but were significantly different for GFAP ( $p=0,0100$, student $t$-test). Open columns, scAAV2/9-CMV-EGFP; solid columns, scAAV2/9-2YF-CMV-EGFP. Data are expressed as

means \pm SD. Analysis was performed using confocal microscopy and counting the number co-labeled cells on five sections per animal $(n=3$ rats for GFP/NeuN, GFP/GFAP, and GFP/Olig2). 
systemic intravenous injection of AAV2/9 was enhanced by capsid tyrosine-to-phenylalanine mutant (Dalkara et al., 2012; Dehay etal., 2012) in a site presumably involved in the ubiquitination and the proteasomal degradation of AAV capsids (Zhong etal., 2008). We injected titer-matched scAAV2/9CMV-EGFP and scAAV2/9-2YF-CMV-EGFP viral suspensions $\left(10^{12} \mathrm{vg} / \mathrm{ml}\right)$ in the cisterna magna of adult female Wistar rats (scAAV2/9, $n=5$; scAAV2/9-2YF, $n=5$ ). One month post-injection, animals were perfused and brains processed for immunohistochemistry. Figure 1 shows the anteroposterior distribution of GFP transgene expression in animals injected with the scAAV2/9-2YF-CMV-EGFP vector. The labeling was present on all coronal sections from the anterior cortex to the cerebellum. The right inset is a magnification of the cerebellar area, showing the typical morphological organization of Purkinje neurons. Interestingly, the density of GFP-positive cells was highest in the regions bordered by the Virchow-Robin subarachnoid space (cortex and cerebellum) and the third ventricle (hippocampus) in which the CSF from the cisterna magna circulates. Figure 2 shows that the density of GFP-positive cells in the cortex (Figures 2A-C) and in the hippocampus (Figures 2D-F) was approximatively fivefold higher after scAAV2/9-2YF-CMV-EGFP (Figures 2B,E) injection as compared to scAAV2/9-CMV-EGFP (Figures 2A,D).

\section{INTRACISTERNAL DELIVERY OF SCAAV2/9 AND sCAAV2/9-2YF USING THE CONSTITUTIVE CMV PROMOTER MEDIATES GENE TRANSFER INTO NEURONS, ASTROCYTES AND OLIGODENDROCYTES}

With both vectors, the majority of cells expressing GFP were neurons (labeled with NeuN marker; respectively, $42.9 \pm 0.0 \%$ and $49.9 \pm 11.8 \%$ for scAAV2/9 and scAAV2/9-2YF, in the




cortex (Figure 3) and $73.7 \pm 6 \%$ and $83.2 \pm 1.8$ in the hippocampus (Figure 4). Astrocytes and oligodendrocytes were also transduced with both vectors. Interestingly, the $2 \mathrm{YF}$ mutation differentially affected the proportion of GFP-positive cells expressing the GFAP astrocytic marker in the hippocampus (25.6 $\pm 6.9 \%$ of the GFP-positive cells for scAAV2/9 versus $6.8 \pm 1.5 \%$ for scAAV2/9-2YF; $p=0.01$; Figure 4 ) but not in the cortex $(31.9 \pm 9.4 \%$ of the GFP-positive cells for scAAV2/9 versus $30.0 \pm 6.9 \%$ for scAAV2/9-2YF; $p=0.7971$; Figure 3).

In contrast, the proportions of GFP-expressing cells which were Olig2-positive oligodendrocytes were not significantly different between both vectors, in both regions (Student $t$-test). However, a tendency for a lower proportion of GFP:Olig2 double labeled cells with the scAAV2/9-2YF mutant was observed in the hippocampus ( $p=0.050$; student $t$-test).
No GFP-positive cells expressed the IbaI marker of microglial cells with both vectors in both regions (data not shown).

\section{INFLAMMATION-INDUCIBLE GENE DELIVERY RESPONSE TO SYSTEMIC KA ADMINISTRATION IN THE CORTEX AND THE HIPPOCAMPUS}

Injection of scAAV2/1-NF8-d1-EGFP into the hippocampal layers followed, 1 month later by intraperitoneal KA injection resulted in a strong transgene expression (Chtarto et al., 2013). However, in order to determine which regions of the brain are affected by the pathology, measured by NFKB activation, a global delivery of the vector is desirable.

In the present study, we have therefore injected scAAV2/9-2YFNF8-d1-EGFP ( $10^{10}$ viral particles in $\left.10 \mu \mathrm{l}\right)$ in the cisterna magna of adult rats. Two weeks later, intraperitoneal injections of KA $(n=8)$ or saline as a control $(n=6)$ were performed. Animals were perfused 2 weeks post-treatment. KA induced a strong
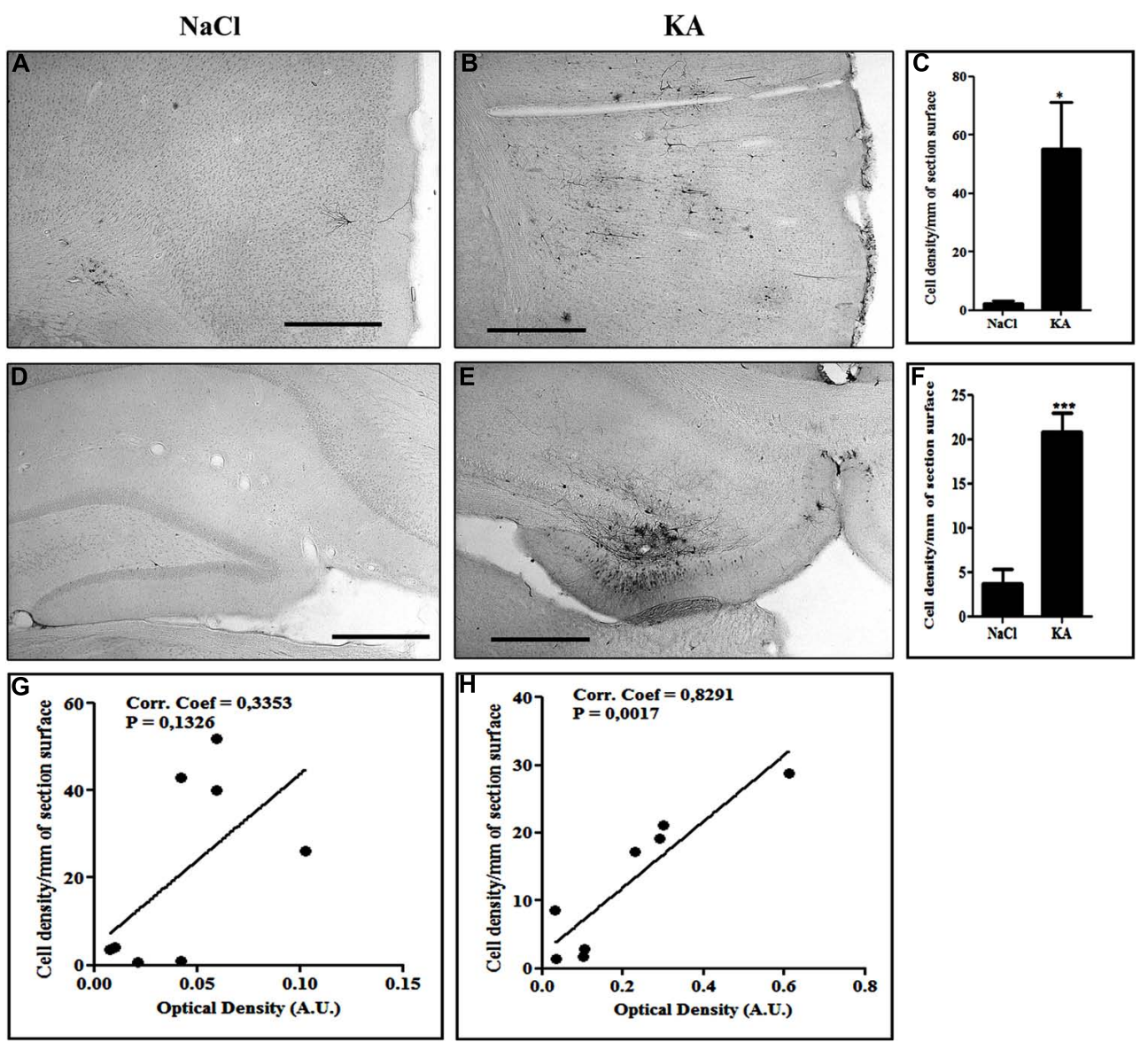

FIGURE 6 | Kainic acid induces NFKB activation-dependent transgene expression. Recombinant scAAV2/9-2YF-NF8-d1-EGFP $\left(10^{10} \mathrm{vg}\right)$ was injected in the cisterna magna of Sprague-Dawley rats. The animals were kept in two groups: one group was intraperitoneally injected with KA ( $n=8$ ) 1 month post virus injection and the other group received only saline $(n=6)$. The animals were sacrificed two weeks after injection. Vibratome brain sections $(50 \mu \mathrm{m})$ were immuno-labeled using anti-GFP antibody. The pictures show representatives examples of GFP-labeling in the cortex $(\mathbf{A}, \mathbf{B})$ and of the hippocampus $(\mathbf{D}, \mathbf{E})$. The cell density in the cortex (C) and in the hippocampus (F) was evaluated as described in
Section "Materials and Methods." Data are expressed as mean \pm SD. The results obtained in the cortex show a significant induction of GFP expression in the KA-treated rats compared to saline treated rats ( $p=0,0207$, student $t$-test). In the hippocampus the cell density in the KA-treated rats is highly increased compared to the saline-treated rats ( $p=0,0005$, student $t$-test). KA, kainic acid; saline, $0.9 \% \mathrm{NaCl}$. The cell density per $\mathrm{mm}^{2}$ of section surface was correlated to the CD11B intensity staining, respectively, in cortex (G; Correlation Coefficient $=0,3353$ and $P=0,1326)$ and hippocampus $(\mathbf{H}$; Correlation Coefficient $=0,8291$ and $p=0,0017$ ). Scale bar: $500 \mu \mathrm{m}$. 
inflammatory response characterized by prominent microglial activation as evidenced by CD11b labeling in the hippocampus and cortex but not in the cerebellum (Figure 5).

We then wanted to analyze whether the regional pattern of GFP transgene expression driven by the NFкB-inducible promoter corresponded to microglial activation. Indeed, Figure 6 shows representatives examples of GFP-labeling in the cortex (Figures 6A,B) and of the hippocampus (Figures 6D,E). The cell density in the cortex (Figure 6C) and in the hippocampus (Figure 6F) was evaluated. The results obtained in the cortex show a significant induction of GFP expression in the KA-treated rats compared to saline treated rats $(p=0.0207$, student $t$-test). In the hippocampus the cell density in the KA-treated rats is highly increased compared to the saline-treated rats $(p=0.0005$, student $t$-test). In contrast, in the untreated group, very few GFPpositive cells were evidenced (see Figures $6 \mathrm{~A}, \mathrm{D}$ ). The intensity of the labeling in treated animals was variable both in terms of surface area and number of cells labeled. This variability could due to variability in the KA treatment efficacy (previously reported) or to variability in the injections, also observed in the AAVCMV group (see Figure 2). The cell density correlates to the intensity of the CD11b staining in the hippocampus (H; Correlation Coefficient $=0.8291$ and $p=0.0017$ ) but not in the cortex (G; Correlation Coefficient $=0.3353$ and $P=0.1326$ ). In addition, no GFP positive cell was evidenced in the cerebellum after scAAV2/9-2YF-NF8-d1-EGFP in the cisterna magna (Figure 7).

We then characterized the cellular specificity of scAAV2/9-2YFNF8-d1-EGFP-mediated transgene expression in KA-treated rats. In both the hippocampus and the cortex, the vast majority of GFPpositive cells were neurons, with a very small percentage of labeled astrocytes (Figure 8) and no oligodendrocyte or microglial cells (data not shown)

\section{DISCUSSION}

Gene delivery to the whole brain has been described following intravenous (Duque et al., 2009; Foust et al., 2009; Mccarty et al., 2009; Fu et al., 2011; Gray et al., 2011) or intra- CSF rAAV administration (Fu et al., 2003, 2010; Samaranch et al., 2012, 2013; Bucher et al., 2013; Gray et al., 2013). Important differences exist in global efficiency between different regions of the brain after intracisternal vector injection, the superficial regions in the immediate vicinity to the cranial sub-arachnoid space being more efficiently transduced than the deeper regions (Samaranch et al., 2012). These data suggest that penetration of the viral particles into deep brain layers is limiting.

Here, we show that intracisternal injection of AAV2/9 leads to gene transfer into neurons, astrocytes, and oligodendrocytes with the highest efficiency in cortex, hippocampus, and cerebellum and a lower efficiency in the striatum, and substantia nigra. Additionally, we show that a rationally designed mutation replacing two surface tyrosine supposedly involved in the recognition by the ubiquitin-proteasome complex, by a phenylalanine residue (Dalkara etal., 2012) enhanced global gene transfer efficiency. Interestingly, the ratio of glial versus neuronal transgene expression was modified by the 2 YF mutation in the hippocampus (but not in the cortex), consistently with previous data obtained after intravenous injection (Dalkara et al., 2012; see Supplementary Figure 3).

Finally, we demonstrate that in response to systemic KA administration, transgene expression from a NFкB-responsive promoter was induced in the hippocampus and the cortex but not in the cerebellum. Our data are consistent with a previously published study showing that the expression of NFкB was increased in the cortex and slightly decreased in the cerebellum following intraperitoneal injections of KA (Rong and Baudry, 1996).

The CSF produced in the choroid plexus is thought to travel to the cisterna magna, then in the Virchow-Robin subarachnoid space and flow into brain parenchyma along cerebral arteries. Molecules dissolved in the CSF are exchanged between perivascular spaces and brain interstitial fluids from which they can penetrate the brain parenchyma through the spaces between astrocytic feet and the basal laminae. These exchanges were suggested to require aquaporin-4 (Iliff et al., 2012; see Figure 9). The brain penetration properties of molecules administered into the cisterna magna varies with the size of the molecules. Small dyes enter the brain by diffusion into the interstitial fluid through gaps between astrocytic feet and the capillaries basal laminae whereas large molecules $(2000 \mathrm{kD})$ similar to AAV particles (MW of approximately $4000 \mathrm{KD}$ ) remain confined in the perivascular spaces. They do not enter the interstitial fluid (Iliff et al., 2012). From the cisterna magna, viral particles are thus expected to circulate in

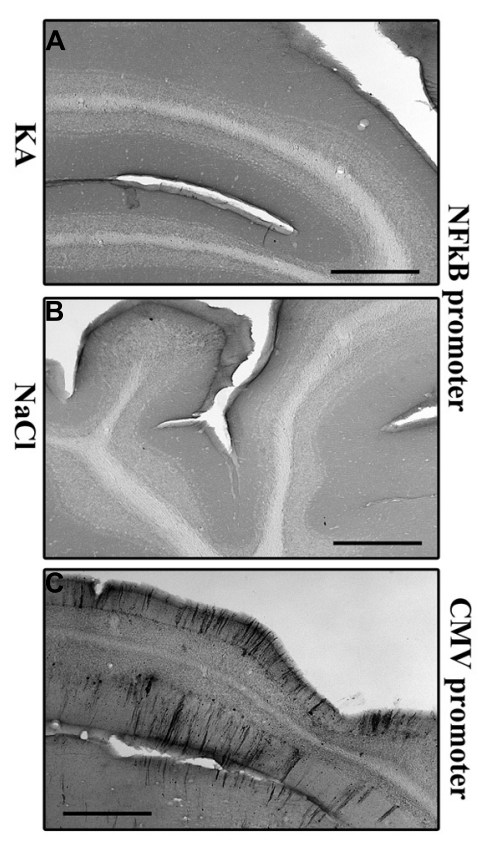

FIGURE 7 | Kainic acid does not induce NFKB activation-mediated transgene expression in the cerebellum. $K A(5 \mathrm{mg} / \mathrm{kg})$ was injected intraperitoneally 5 weeks after intracisternal injection of scAAV2/9-2YF-NF8-d1-EGFP. Fifty $\mu \mathrm{m}$ coronal sections were labeled with GFP antibodies. Scale bar: $500 \mu \mathrm{m}$. The GFP staining is almost inexistent in $\mathrm{KA}$ - and saline-treated rats injected with scAAV2/9-2YF-NF8-d1-EGFP (A,B) while a strong GFP staining was demonstrated in rats injected with scAAV2/9-2YF-CMV-EGFP (C) 


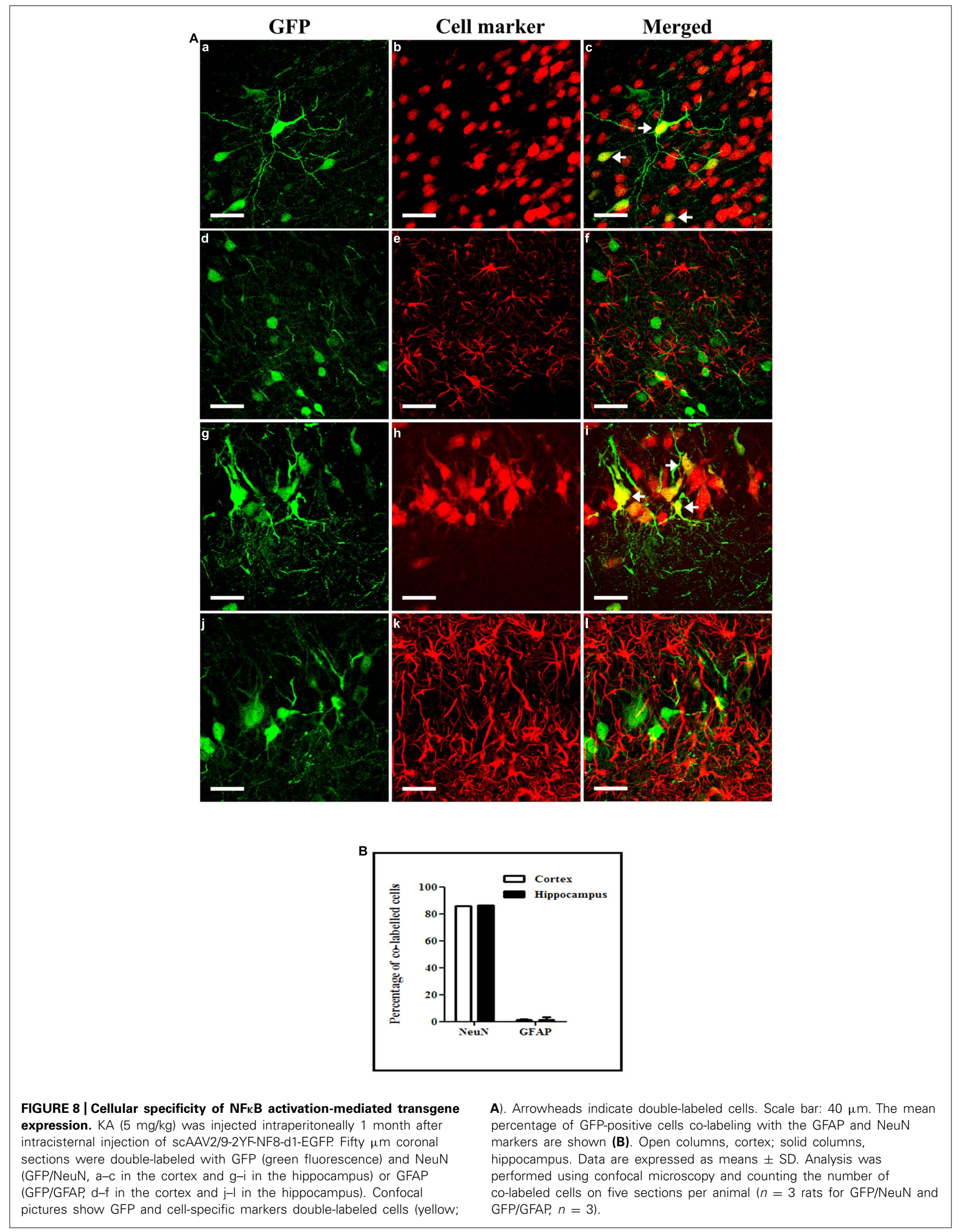


the Virchow-Robin subarachnoid space and enter the brain along spaces around penetrating blood vessels. The mechanism by which AAV particles, enter the brain interstitial fluid is unknown. The fact that AAV2 particles transduce the brain after intracisternal delivery (Fu et al., 2003) exclusively in the presence of mannitol, suggests that the up-regulation of expression of aquaporin 4 by mannitol (Mccarty et al., 2009; Fu et al., 2010) may play a role of the entry of particles (Arima et al., 2003). In contrast, cisternally injected AAV2/9 particles enter the brain regardless of the presence of mannitol or sorbitol. However, the use of sorbitol (Gray et al., 2011) or mannitol (Bockstael et al., unpublished observations) enhanced transduction efficiency. Mannitol also enhanced trans-brain-blood barrier gene delivery mediated by AAV2/9 (Fu et al., 2011). Altogether these data suggest that the mechanism of viral particles entry into the brain parenchyma after intravascular and intra-CSF administration might share at least a common pathway involving the aquaporin 4 water channel.

Compared to intravenous injections, injections into the CSF were shown to reduce the bio-distribution of vectors into peripheral organs (Gray et al., 2013) as well as avoiding the inhibitory effect on gene transfer mediated by anti-AAV neutralizing antibodies (Gray et al., 2013). However, a strong inflammatory response to non-self-transgene products and elimination of transduction was observed in primates (Samaranch et al., 2014). This can be explained by the presence of activated $\mathrm{T}$ secreting cytokines at the choroid plexus-brain interface which can in some circumstances enter the CNS (Schwartz and Baruch, 2014).

We have previously established a proof-of-concept for diseaseinducible gene delivery in the brain using an AAV vector responding to $\mathrm{NFKB}$ activation as a probe for inflammatory responses (Chtarto et al., 2013). In this study, the reporter vector was injected focally in the parenchyma prior to a systemic KA injection and mediated inducible and specific transgene expression in the hippocampus but not in the cerebellum, regions, respectively, known to be affected or spared by the pathology (Rong and Baudry, 1996). However, such a focal approach relies on the knowledge of the mechanism of disease induction/progression in the animal model.

Vector delivery in the CSF allows to reveal the pathology as well as to deliver therapeutic factors in several affected brain regions after a single vector injection and in a disease-regulated manner. However, in order to reflect the regional specificity of the disease, the distribution of the transgene product should be homogenous throughout the brain. In our study, the distribution of transgene expression is heterogeneous, with a higher efficiency in cerebellum, cortex, and hippocampus and a limited efficiency in deeper regions such as striatum. Methods to stimulate the perivascular pump or increase the functionality of water channels at the astrocytic feet could enhance viral particle penetration into the brain.

In other recent studies, the model of CSF circulation has been challenged and it has proposed that CSF is reabsorbed into brain capillaries (Pardridge, 2011). Thus AAV2/9 viral particles might first enter the brain capillaries than subsequently enter into the brain parenchyma through the blood-brain-barrier.

\section{AUTHOR CONTRIBUTIONS}

Conceived and designed the experiments: Abdelwahed Chtarto, Liliane Tenenbaum, Olivier De Witte, Marc Levivier.

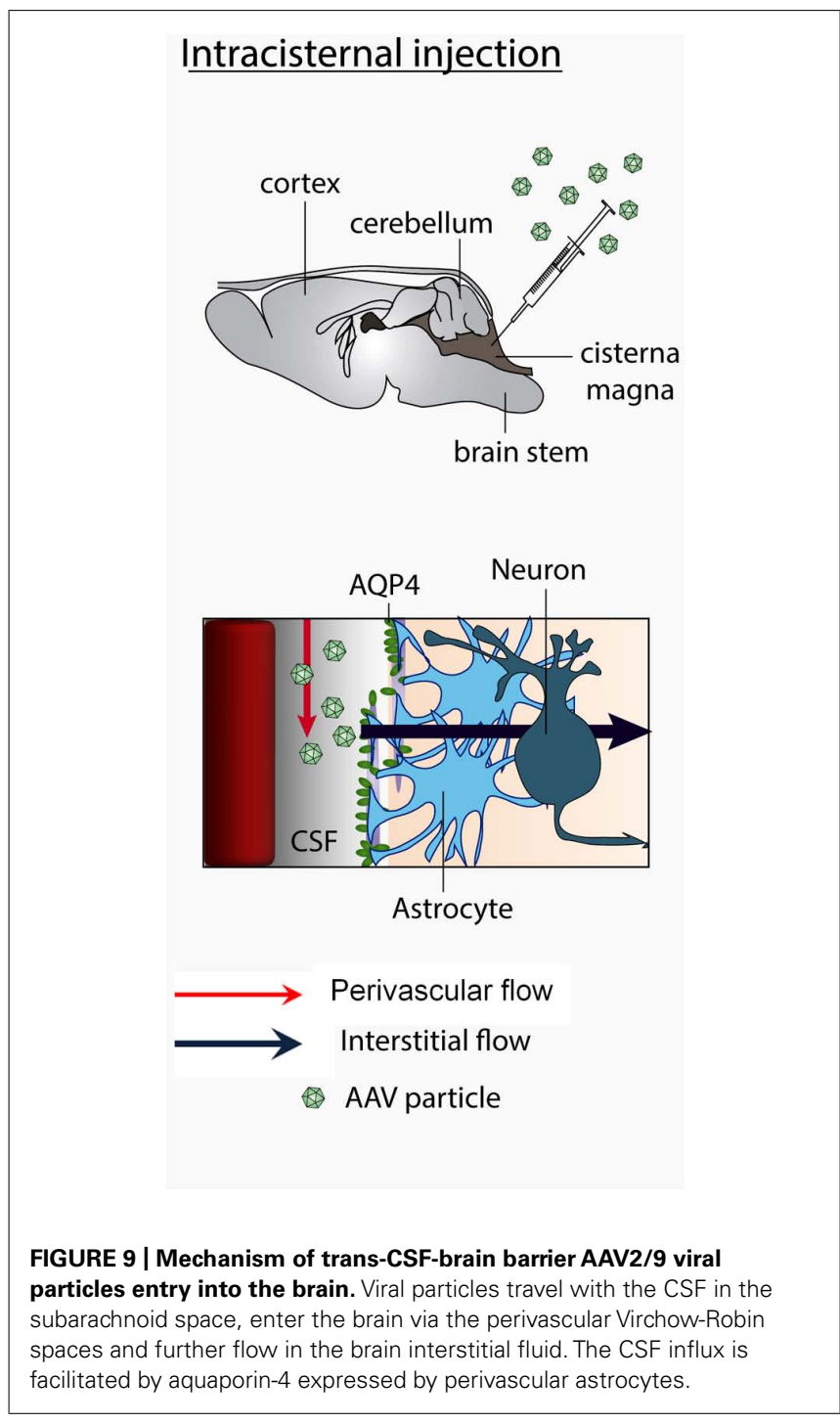

Performed the experiments: Olivier Bockstael, Abdelwahed Chtarto, Catherine Melas. Analyzed the data: Olivier Bockstael, Abdelwahed Chtarto. Contributed reagents/materials/analysis tools: Olivier Bockstael, Abdelwahed Chtarto, Deniz Dalkara, Liliane Tenenbaum. Wrote the paper: Abdelwahed Chtarto, Liliane Tenenbaum, Olivier Bockstael.

\section{ACKNOWLEDGMENTS}

We thank Dr. Frederic Bollet-Quivogne for the quantification of GFP-expression and Professor Ilse Smolders for discussions and advice concerning the KA treatment. This work was supported by Fonds d'Encouragement à la Recherche from the Faculty of Medicine of the Université Libre de Bruxelles (FER 2014), Swiss National Research Foundation (FNS; grant number: FN31003A127177) and EU FP7 Marie Curie IAPP BrainVectors (contract $n^{\circ}$. 286071).

\section{REFERENCES}

Arima, H., Yamamoto, N., Sobue, K., Umenishi, F., Tada, T., Katsuya, H., et al. (2003). Hyperosmolar mannitol simulates expression of aquaporins 4 and 9 through a 
p38 mitogen-activated protein kinase-dependent pathway in rat astrocytes. J. Biol. Chem. 278, 44525-44534. doi: 10.1074/jbc.M304368200

Bockstael, O., Melas, C., Pythoud, C., Levivier, M., McCarty, D., Samulski, R. J., et al. (2012). Rapid transgene expression in multiple precursor cell types of adult rat subventricular zone mediated by adeno-associated type 1 vectors. Hum. Gene Ther. 23, 742-753. doi: 10.1089/hum.2011.216

Brown, G. C., and Neher, J. J. (2014). Microglial phagocytosis of live neurons. Nat. Rev. Neurosci. 15, 209-216. doi: 10.1038/nrn3710

Bucher, T., Colle, M. A., Wakeling, E., Dubreil, L., Fyfe, J., Briot-Nivard, D., et al. (2013). scAAV2/9 intracisternal delivery results in efficient gene transfer to the central nervous system of a feline model of motor neuron disease. Hum. Gene Ther. 24, 670-682. doi: 10.1089/hum.2012.218

Chtarto, A., Bockstael, O., Gebara, E., Vermoesen, K., Melas, C., Pythoud, C., et al. (2013). An adeno-associated virus-based intracellular sensor of pathological nuclear factor-kappaB activation for disease-inducible gene transfer. PLoS ONE 8:e53156. doi: 10.1371/journal.pone.0053156

Dalkara, D., Byrne, L. C., Lee, T., Hoffmann, N. V., Schaffer, D. V., and Flannery, J. G. (2012). Enhanced gene delivery to the neonatal retina through systemic administration of tyrosine-mutated AAV2/9. Gene Ther. 19, 176-181. doi: 10.1038/gt.2011.163

Dehay, B., Dalkara, D., Dovero, S., Li, Q., and Bezard, E. (2012). Systemic scAAV2/9 variant mediates brain transduction in newborn rhesus macaques. Sci. Rep. 2:253. doi: 10.1038/srep00253

Dexter, D. T., and Jenner, P. (2013). Parkinson disease: from pathology to molecular disease mechanisms. Free Radic. Biol. Med. 62, 132-144. doi: 10.1016/j.freeradbiomed.2013.01.018

Duque, S., Joussemet, B., Riviere, C., Marais, T., Dubreil, L., Douar, A. M., et al. (2009). Intravenous administration of self-complementary AAV2/9 enables transgene delivery to adult motor neurons. Mol. Ther. 17, 1187-1196. doi: $10.1038 / \mathrm{mt} .2009 .71$

Foust, K. D., Nurre, E., Montgomery, C. L., Hernandez, A., Chan, C. M., and Kaspar, B. K. (2009). Intravascular AAV2/9 preferentially targets neonatal neurons and adult astrocytes. Nat. Biotechnol. 27, 59-65. doi: 10.1038/nbt.1515

Fu, H., Dirosario, J., Kang, L., Muenzer, J., and Mccarty, D. M. (2010). Restoration of central nervous system alpha- $\mathrm{N}$-acetylglucosaminidase activity and therapeutic benefits in mucopolysaccharidosis IIIB mice by a single intracisternal recombinant adeno-associated viral type 2 vector delivery. J. Gene Med. 12, 624-633. doi: 10.1002/jgm. 1480

Fu, H., Dirosario, J., Killedar, S., Zaraspe, K., and Mccarty, D. M. (2011). Correction of neurological disease of mucopolysaccharidosis IIIB in adult mice by rAAV2/9 trans-blood-brain barrier gene delivery. Mol. Ther. 19, 1025-1033. doi: 10.1038/mt.2011.34

Fu, H., Muenzer, J., Samulski, R. J., Breese, G., Sifford, J., Zeng, X., et al. (2003). Selfcomplementary adeno-associated virus serotype 2 vector: global distribution and broad dispersion of AAV-mediated transgene expression in mouse brain. Mol. Ther. 8, 911-917. doi: 10.1016/j.ymthe.2003.08.021

Gray, S. J., Matagne, V., Bachaboina, L., Yadav, S., Ojeda, S. R., and Samulski, R. J. (2011). Preclinical differences of intravascular AAV2/9 delivery to neurons and glia: a comparative study of adult mice and nonhuman primates. Mol. Ther. 19, 1058-1069. doi: 10.1038/mt.2011.72

Gray, S. J., Nagabhushan, K. S., McCown, T. J., and Jude, S. R. (2013). Global CNS gene delivery and evasion of anti-AAV-neutralizing antibodies by intrathecal AAV administration in non-human primates. Gene Ther. 20, 450-459. doi: 10.1038/gt.2012.101

Iliff, J. J., Wang, M., Liao, Y., Plogg, B. A., Peng, W., Gundersen, G. A., et al. (2012). A perivascular pathway facilitates CSF flow through the brain parenchyma and the clearance of interstitial solutes, including amyloid beta. Sci. Transl. Med. 4:147ra111. doi: 10.1126/scitranslmed.3003748

Liu, L., and Duff, K. (2008). A technique for serial collection of cerebrospinal fluid from the cisterna magna in mouse. J. Vis. Exp. 21, 960. doi: 10.3791/960

Lock, M., McGorray, S., Auricchio, A., Ayuso, E., Beecham, E. J., Blouin-Tavel, V., et al. (2010). Characterization of a recombinant adeno-associated virus type 2 Reference Standard Material. Hum. Gene Ther. 21, 1273-1285. doi: 10.1089/hum.2009.223

London, A., Cohen, M., and Schwartz, M. (2013). Microglia and monocytederived macrophages: functionally distinct populations that act in concert in
CNS plasticity and repair. Front. Cell. Neurosci. 7:34. doi: 10.3389/fncel.2013. 00034

Mccarty, D. M., Dirosario, J., Gulaid, K., Muenzer, J., and Fu, H. (2009). Mannitol-facilitated CNS entry of rAAV2 vector significantly delayed the neurological disease progression in MPS IIIB mice. Gene Ther. 16, 1340-1352. doi: 10.1038/gt.2009.85

Mccarty, D. M., Fu, H., Monahan, P. E., Toulson, C. E., Naik, P., and Samulski, R. J. (2003). Adeno-associated virus terminal repeat (TR) mutant generates selfcomplementary vectors to overcome the rate-limiting step to transduction in vivo. Gene Ther. 10, 2112-2118. doi: 10.1038/sj.gt.3302134

Pardridge, W. M. (2011). Drug transport in brain via the cerebrospinal fluid. Fluids Barriers CNS 8:7. doi: 10.1186/2045-8118-8-7

Rong, Y., and Baudry, M. (1996). Seizure activity results in a rapid induction of nuclear factor-kappa $B$ in adult but not juvenile rat limbic structures. J. Neurochem. 67, 662-668. doi: 10.1046/j.1471-4159.1996.670 20662.x

Samaranch, L., Salegio, E. A., San, S. W., Kells, A. P., Bringas, J. R., Forsayeth, J., et al. (2013). Strong cortical and spinal cord transduction after AAV7 and AAV2/9 delivery into the cerebrospinal fluid of nonhuman primates. Hum. Gene Ther. 24, 526-532. doi: 10.1089/hum.2013.005

Samaranch, L., Salegio, E. A., San, S. W., Kells, A. P., Foust, K. D., Bringas, J. R., et al. (2012). Adeno-associated virus serotype 9 transduction in the central nervous system of nonhuman primates. Hum. Gene Ther. 23, 382-389. doi: 10.1089/hum.2011.200

Samaranch, L., San, S. W., Kells, A. P., Salegio, E. A., Heller, G., Bringas, J. R., et al. (2014). AAV2/9-mediated expression of a non-self protein in nonhuman primate central nervous system triggers widespread neuroinflammation driven by antigen-presenting cell transduction. Mol. Ther. 22, 329-337. doi: 10.1038/mt.2013.266

Schwartz, M., and Baruch, K. (2014). The resolution of neuroinflammation in neurodegeneration: leukocyte recruitment via the choroid plexus. EMBO J. 33, 7-22. doi: 10.1002/embj.201386609

Somera-Molina, K. C., Nair, S., Van Eldik, L. J., Watterson, D. M., and Wainwright, M. S. (2009). Enhanced microglial activation and proinflammatory cytokine upregulation are linked to increased susceptibility to seizures and neurologic injury in a two-hit' seizure model. Brain Res. 1282, 162-172. doi: 10.1016/j.brainres.2009.05.073

Vermoesen, K., Smolders, I., Massie, A., Michotte, Y., and Clinckers, R. (2010). The control of kainic acid-induced status epilepticus. Epilepsy Res. 90, 164-166. doi: 10.1016/j.eplepsyres.2010.04.001

Williams, P. A., White, A. M., Clark, S., Ferraro, D. J., and Swiercz, W. (2009). Development of spontaneous recurrent seizures after kainate-induced status epilepticus. J. Neurosci. 29, 2103-2112. doi: 10.1523/JNEUROSCI.0980-08.2009

Zhong, L., Li, B., Jayandharan, G., Mah, C. S., Govindasamy, L., Agbandje-McKenna, M., et al. (2008). Tyrosine-phosphorylation of AAV2 vectors and its consequences on viral intracellular trafficking and transgene expression. Virology 381, 194-202. doi: 10.1016/j.virol.2008.08.027

Conflict of Interest Statement: The authors declare that the research was conducted in the absence of any commercial or financial relationships that could be construed as a potential conflict of interest.

Received: 20 August 2014; accepted: 04 November 2014; published online: 02 December 2014.

Citation: Bockstael O, Tenenbaum L, Dalkara D, Melas C, De Witte O, Levivier M and Chtarto A (2014) Intracisternal delivery of NFK B-inducible scAAV2/9 reveals locoregional neuroinflammation induced by systemic kainic acid treatment. Front. Mol. Neurosci. 7:92. doi: 10.3389/fnmol.2014.00092

This article was submitted to the journal Frontiers in Molecular Neuroscience. Copyright (C) 2014 Bockstael, Tenenbaum, Dalkara, Melas, De Witte, Levivier and Chtarto. This is an open-access article distributed under the terms of the Creative Commons Attribution License (CC BY). The use, distribution or reproduction in other forums is permitted, provided the original author(s) or licensor are credited and that the original publication in this journal is cited, in accordance with accepted academic practice. No use, distribution or reproduction is permitted which does not comply with these terms. 